\title{
Criminologie
}

\section{Psychopathie et lien avec la victime chez les agresseurs sexuels de femmes adultes}

\section{Normand Aubertin et Gilles Côté}

Volume 38, numéro 1, printemps 2005

Filles et déviance : perspectives développementales

URI : https://id.erudit.org/iderudit/011490ar

DOI : https://doi.org/10.7202/011490ar

Aller au sommaire du numéro

\section{Éditeur(s)}

Les Presses de l'Université de Montréal

ISSN

0316-0041 (imprimé)

1492-1367 (numérique)

Découvrir la revue

Citer cet article

Aubertin, N. \& Côté, G. (2005). Psychopathie et lien avec la victime chez les agresseurs sexuels de femmes adultes. Criminologie, 38(1), 189-206.

https://doi.org/10.7202/011490ar
Résumé de l'article

La psychopathie est un phénomène peu étudié chez les agresseurs sexuels des conjointes. L'hypothèse que les caractéristiques de ces agresseurs sexuels tiennent d'un mode de fonctionnement psychopathique, par opposition aux agresseurs sexuels de femmes sans lien d'intimité, a donc été étudiée. Pour ce faire, 27 agresseurs sexuels de conjointes et 70 agresseurs sexuels de personnes qui ne sont pas leurs conjointes furent comparés en utilisant la Psychopathy Checklist Revised (PCL-R). Dans l'ensemble, cinq sujets seulement ont rempli les critères de la psychopathie. Sur la base catégorielle, plus d'agresseurs sexuels de conjointes se retrouvent parmi les cas mixtes, les agresseurs sexuels de non-conjointes se retrouvant plus souvent parmi les non-psychopathes. Sur la base d'un continuum, les agresseurs sexuels de conjointes ont cependant obtenu un score moyen général plus élevé à la PCL-R.
Ce document est protégé par la loi sur le droit d'auteur. L'utilisation des services d’Érudit (y compris la reproduction) est assujettie à sa politique d'utilisation que vous pouvez consulter en ligne.

https://apropos.erudit.org/fr/usagers/politique-dutilisation/ 


\title{
Psychopathie et lien avec la victime chez les agresseurs sexuels de femmes adultes
}

\author{
Normand Aubertin \\ Université du Québec à Trois-Rivières \\ Institut Philippe Pinel de Montréal \\ n.aubertin@videotron.ca \\ Gilles Côté \\ Université du Québec à Trois-Rivières \\ Institut Philippe Pinel de Montréal \\ gilles_cote@uqtr.uquebec.ca
}

RÉSUMÉ - La psychopathie est un phénomène peu étudié chez les agresseurs sexuels des conjointes. L'hypothèse que les caractéristiques de ces agresseurs sexuels tiennent d'un mode de fonctionnement psychopathique, par opposition aux agresseurs sexuels de femmes sans lien d'intimité, a donc été étudiée. Pour ce faire, 27 agresseurs sexuels de conjointes et 70 agresseurs sexuels de personnes qui ne sont pas leurs conjointes furent comparés en utilisant la Psychopathy Checklist Revised (PCLR). Dans l'ensemble, cinq sujets seulement ont rempli les critères de la psychopathie. Sur la base catégorielle, plus d'agresseurs sexuels de conjointes se retrouvent parmi les cas mixtes, les agresseurs sexuels de non-conjointes se retrouvant plus souvent parmi les non-psychopathes. Sur la base d'un continuum, les agresseurs sexuels de conjointes ont cependant obtenu un score moyen général plus élevé à la PCL-R.

ABSTRACT - Psychopathy has rarely been studied in the wife rapist population. The hypothesis of our study was that the characteristics of the spouse sexual offenders refer more to a psychopatic functioning than the non-spouse sexual offenders. A sample of 27 spouse sexual offenders and 70 non-spouse sexual offenders was selected. The «Psychopathy Checklist Revised» (PCL-R) was used to evaluate psychopathy. The results were that only five subjects meet the criterion of psychopathy in the whole sample. On a categorial basis, difference appeared in the distribution of the different samples among the cases categorised as "mixed" and the "non-psychopaths", the spouse sexual offenders being more often diagnosed as "mixed" and the non-spouse 
sexual offenders being more often diagnosed as "non-psychopaths". On a dimensional basis, the mean score at the PCL-R of the spouse sexual offenders was significantly higher than the non-spouse sexual offenders.

\section{État du problème}

Le phénomène de la violence conjugale fait l'objet d'une attention particulière dans la société canadienne. Des programmes de sensibilisation et de traitement ont été élaborés ces dernières années, que ce soit dans la communauté ou dans le milieu institutionnel (Rondeau, 1988; Comité canadien sur la violence faite aux femmes, 1993; Kropp et al., 1995; Stewart et al., 1999). Parmi les conjoints violents, certains vont agresser sexuellement leur conjointe. Huit pour cent des femmes au Canada mentionnent avoir été agressées sexuellement par un conjoint (Centre canadien de la statistique juridique, 1994). L'agression sexuelle de la conjointe, passée ou présente, présente un facteur important de risques de récidive (Walker, 1979; Sonkin, 1987; Stuart et Campbell, 1989; Goldsmith, 1990; Saunders, 1992; Kropp et al., 1995); il y a donc lieu de chercher à mieux comprendre le sous-groupe des violeurs de conjointe. Ces derniers se trouvent au carrefour de diverses problématiques en ce qui a trait à l'évaluation du risque de comportements violents. Faut-il comprendre cette problématique sous l'angle de la violence conjugale, de la déviance sexuelle ou, encore, d'un fonctionnement purement psychopathique?

\section{Violence familiale et psychopathie}

Huss et Langhinrichsen-Rohling (2000) mentionnent que, bien que les psychopathes ne représentent pas la majorité des agresseurs de conjointes, la littérature scientifique sur la psychopathie suggère qu'ils pourraient constituer une minorité importante (15 à 30\%). Caesar (1986) a utilisé le MMPI pour déterminer trois groupes d'agresseurs de conjointes: l'«altruiste non révélé» généralement dépendant et peu sûr de lui; le «sauveteur révélé» présentant des traits de personnalité histrionique; le «tyran» caractérisé par des traits psychopathiques et paranoïdes. La distribution des sujets à l'intérieur de chaque groupe ne fut pas établie.

Gondolf (1988) définit également trois groupes d'agresseurs de conjointes. Deux de ces groupes, les «sociopathes/psychopathes» (environ $8 \%$ de la population cible) et les «antisociaux» (environ $42 \%$ de la 
population cible) sont surtout caractérisés par l'abus sexuel, la violence envers les enfants et le comportement antisocial. Le troisième groupe, les «agresseurs typiques» (environ $50 \%$ de la population cible) sont moins abusifs que les deux autres groupes aux plans physique, verbal et sexuel. Les auteurs ont utilisé l'histoire sociale et des données comportementales comme variables de base dans leur analyse de groupement (cluster). Par la suite, ils ont demandé à une équipe de cliniciens d'interpréter et d'établir les regroupements. Toutefois, il faut souligner que la définition de «sociopathes/psychopathes» utilisée pour désigner le premier groupe n'est pas précisée et qu'aucun outil diagnostique ne fut utilisé.

Saunders (1992) a incorporé six (6) variables dans son étude (c.-à-d. dépression, colère, violence généralisée, sévérité de la violence conjugale, attitude envers les femmes et intoxication au moment des incidents de violence). Trois sous-groupes sont ressortis, soit les agresseurs qui s'attaquent uniquement à la famille ( $52 \%$ de l'échantillon), les agresseurs généralement violents $(29 \%)$ et les agresseurs instables émotivement (19\%). Les agresseurs généralement violents sont caractérisés par une violence extra et intrafamiliale plus importante. Leur violence est associée à la consommation d'alcool. Ils rapportent des niveaux de colère et de dépression variant de faible à modéré. Ces caractéristiques laissent croire que les psychopathes devraient être classifiés dans le sous-groupe des agresseurs généralement violents.

La notion d'intensité émotionnelle a été à l'origine de l'étude de Gottman et al. (1995). Ceux-ci ont proposé une nouvelle typologie d'agresseurs de conjointes basée sur le rythme cardiaque durant le visionnement vidéo d'un conflit matrimonial. Ainsi, le rythme cardiaque du type I diminue durant l'incident observé, tandis que celui du type II augmente ou reste stable. Selon eux, la diminution du rythme cardiaque pourrait être due à des caractéristiques psychopathiques constitutionnelles, les psychopathes étant caractérisés par un déficit émotionnel. Ces derniers seraient donc moins réactionnels dans des situations riches en émotions; ils réagiraient plutôt à des protoémotions, telle la vengeance, plutôt qu'à la colère. Toutefois, ce point de vue a été passablement remis en question par Hare (1998) qui préfere parler de déficit cognitif dans l'interprétation des situations plutôt que dans la possibilité de ressentir les émotions.

Hale et al. (1988) ont déterminé, en utilisant le MMPI, que $75 \%$ des conjoints violents avaient un score élevé aux échelles de dépression et de psychopathie. Flournoy et Wilson (1991), reproduisant la recherche 
de Hale et al. (1988), obtiennent un pourcentage de $44 \%$ pour le même groupe. Huss et al. (1997) en arrivent à des conclusions similaires, alors que $39 \%$ des cas affichent une élévation significative à l'échelle de déviance psychopathique.

À partir d'une revue de la littérature scientifique, Holtzworth-Munroe et Stuart (1994) ont établi une typologie d'agresseurs de conjointes comprenant trois groupes. Il y a ceux qui s'attaquent uniquement aux membres de la famille (environ $50 \%$ des agresseurs), les agresseurs «dysphoriques/états-limites» (environ $25 \%$ des agresseurs) et les agresseurs «généralement violents/antisociaux» (environ $25 \%$ des agresseurs). Ce dernier groupe est surtout caractérisé par une violence d'intensité modérée à élevée, incluant la violence psychologique et l'abus sexuel. Les individus de ce dernier groupe manifestent plus de violence extrafamiliale; ils ont également une histoire criminelle plus importante. Les auteurs mentionnent y retrouver un plus haut taux de psychopathie, sans toutefois définir clairement ce syndrome. Soulignons enfin que la prévalence rapportée n'est qu'une estimation basée sur les recherches recensées par les auteurs.

Ces études sont basées sur diverses définitions de la psychopathie, ainsi que sur des mesures variées (MMPI ou évaluation par cliniciens). Dans ce contexte, il devient donc difficile de déterminer avec justesse la prévalence de la psychopathie chez les agresseurs de conjointes.

\section{La Psychopathy Checklist}

Actuellement, l'outil le plus utilisé par les chercheurs pour évaluer la psychopathie (Cooke et al., 1998) est la Psychopatby Checklist (Hare, 1980 ; 1985 ; 1991), aussi désignée sous son acronyme le plus récent par suite de la révision de 1991, soit PCL-R. Dans cette échelle, le diagnostic est le résultat d'une cotation de 20 items en trois points. La cote zéro est attribuée à un item qui ne s'applique pas au sujet, la cote un est accordée lorsque les caractéristiques de l'item s'appliquent au sujet jusqu'à un certain point, mais pas suffisamment pour lui valoir la cote deux, laquelle est attribuée lorsqu'il y a une correspondance raisonnablement bonne dans la plupart des aspects les plus essentiels. Le score total peut donc varier entre 0 et 40 . Des valeurs critiques ont été définies pour mieux préciser un mode d'organisation. Ainsi, un score de 30 ou plus permet de poser un diagnostic de psychopathie. Son 
absence appelle un score inférieur à 20, tandis que des résultats entre 20 et 29 renvoient à une problématique dite «mixte». Ces intervalles sont ceux qui permettent la meilleure classification des sujets (Hare, 1991). Par contre, comme la psychopathie est considérée par certains comme une extension de la personnalité normale (Widiger, 1998), la PCL-R est aussi interprétée, à partir du continuum des résultats, dans son aspect dimensionnel.

Des analyses factorielles et des analyses d'items ont permis de faire ressortir deux facteurs (Harpur et al., 1988; Harpur et al., 1989; Cooke et Michie, 1997). Le premier est caractérisé par des traits de personnalité déterminés (narcissiques) et le deuxième par des comportements antisociaux. C'est d'ailleurs le premier facteur qui différencie le diagnostic de psychopathie, selon la PCL-R, du diagnostic de personnalité antisociale du Diagnostic and Statistical Manual of mental disorders (DSM) (American Psychiatric Association, 1994). Le trouble de personnalité antisociale se rapporte davantage à des comportements antisociaux et non à des traits de personnalité. Il est à noter que le facteur 1 (traits de personnalité) est associé à la récidive violente, tandis que le facteur 2 (comportements antisociaux) est associé à la récidive générale (Serin, 1996).

Certaines analyses récentes font plutôt ressortir trois facteurs à l'intérieur de la PCL-R (Cooke et Michie, 2001). Ce modèle hiérarchique à trois facteurs renvoie au «style interpersonnel arrogant et fourbe», à la «vie affective pauvre» et au «comportement impulsif et irresponsable».

\section{Agressions sexuelles et psychopathie}

La prévalence de la psychopathie chez les délinquants sexuels est faible, particulièrement chez les pédophiles, soit entre $3 \%$ et $15 \%$ (Miller et al., 1994; voir aussi Serin et al., 1994; Brown et Forth, 1997; Hart et Hare, 1998; Rochefort et Earls, 1998). Par contre, ces taux sont plus élevés chez les violeurs, la prévalence variant de $35 \%$ à $77 \%$ (Miller et al., 1994; voir également Brown et Forth, 1997; Hart et Hare, 1998).

Par ailleurs, la psychopathie est particulièrement manifeste chez les agresseurs sexuels les plus chroniques et les plus violents (Rice et al., 1990). Comme les agressions sexuelles de conjointes sont généralement plus violentes que les agressions sexuelles de non-conjointes (Groth et Birnbaum, 1979; Koss et al., 1988; St-Yves et al., document 
non publié), la prévalence de la psychopathie ne serait-elle pas plus élevée parmi les agresseurs sexuels de conjointes?

Diverses typologies d'agresseurs sexuels ont été bâties. Barbaree et al. (1994) distinguent quatre types de violeurs. Deux ont une motivation sexuelle, distinction étant faite sur la base d'une composante sadique ou non sadique, et deux ont une motivation agressive, distinction étant faite entre le viol opportuniste et le viol vindicatif. La PCL-R ne permet pas de différencier ces quatre groupes, bien que le facteur 2 (comportements antisociaux) soit statistiquement plus élevé chez les sujets à motivation sexuelle sadique que chez les trois autres sous-types. Le viol vindicatif semble s'apparenter au viol de la conjointe, viol qui a pour motivation l'humiliation et la vengeance, plutôt que les fantaisies sexuelles (St-Yves et al., document non publié).

Hall et Hirschman (1991) ont émis une théorie selon laquelle le viol résulterait de l'interaction de quatre facteurs, un seul étant le précurseur primaire du délit. Ces facteurs sont: 1) l'excitation sexuelle pour le viol 2) les distorsions cognitives justifiant le viol 3) l'impulsivité émotionnelle épisodique et 4) les troubles de la personnalité. Bien que cela ne soit pas exclusif, les psychopathes appartiendraient davantage aux deux dernières catégories par leurs caractéristiques psychologiques, leur niveau de violence générale et le modus operandi de l'agression sexuelle. Les agresseurs sexuels de conjointes, quant à eux, se retrouvent davantage dans les trois dernières catégories, puisqu'ils présentent moins de fantaisies sexuelles déviantes, mais font preuve de plus de distorsions cognitives concernant le viol que les agresseurs sexuels sans lien d'intimité (St-Yves et al., document non publié). Les violeurs conjugaux présenteraient donc plus de traits psychopatiques.

Pour Knight et Prentky (1991), les agresseurs sexuels peuvent être distingués selon la motivation première du délit. Il y a le type opportuniste, généralement impulsif, qui utilise un certain degré de violence, uniquement pour vaincre la résistance de sa victime; celui-ci planifie peu son crime. Le type chroniquement colérique agit sous le coup de la colère et inflige souvent des blessures graves à sa victime. Le type sexuel agit en fonction de fantaisies sexuelles déviantes, sadiques ou non; il présente une série de distorsions cognitives à propos des femmes et de la sexualité. Le type vindicatif nourrit une colère particulière contre les femmes; il agit habituellement avec brutalité dans le but de les humilier. Travaillant à partir de cette typologie et de la conception de la psychopathie définie par la PCL-R, Brown et Forth (1997) ont établi que les 
psychopathes se classent deux fois plus souvent dans les sous-groupes d'agresseurs « opportunistes» $(\mathrm{P}=52,4$ et $\mathrm{NP}=25,6)$ et « envahis par la colère » $(\mathrm{P}=28,6$ et $\mathrm{NP}=15,4)$; que les non-psychopathes sont trois fois plus nombreux à présenter une motivation sexuelle non-sadique $(\mathrm{P}=9,5$ et $\mathrm{NP}=30,8)$; et que peu de psychopathes sont désignés comme agresseurs «vindicatifs» $(\mathrm{P}=0)$. Ces auteures font ressortir que le type «opportuniste» présente un score moyen plus élevé au facteur 1 (facteur relié à la récidive violente selon Serin, 1996) que le type «vindicatif». Les deux types obtiennent des scores équivalents au facteur 2. Comme les violeurs conjugaux se retrouvent plus souvent dans la catégorie des agresseurs «vindicatifs» (St-Yves et al., document non publié), ceci porterait à croire qu'il y a moins de psychopathes parmi les agresseurs sexuels de conjointes. Cependant, il faut interpréter cette conclusion avec prudence puisque les violeurs conjugaux sont peu nombreux. Ils peuvent donc présenter un taux élevé de psychopathie même si l'ensemble des agresseurs «vindicatifs» ont une prévalence plus faible de psychopathie que les agresseurs «opportunistes». Les agresseurs sexuels de conjointes pourraient donc représenter une population particulière parmi les agresseurs «vindicatifs». En somme, ce sous-groupe serait possiblement sousreprésenté dans le groupe des agresseurs «vindicatifs».

Groth et Birnbaum (1979) soutiennent que les agresseurs sexuels sont motivés par trois facteurs, soit le pouvoir (power rape), la colère (anger rape) ou la sexualité. L'agression sexuelle de la conjointe s'apparente à une motivation colérique (anger rape) où le passage à l'acte est impulsif et physiquement brutal. Elle a comme objectif d'humilier la victime. Ces sujets rapportent peu de fantaisies sexuelles déviantes. Les psychopathes devraient se retrouver dans les deux premières catégories, puisque leur passage à l'acte n'a pas une motivation sexuelle (Brown et Forth, 1997). Toutefois, le viol motivé par la colère s'apparente aux agressions sexuelles «vindicatives» de Knight et Prentky (1991), puisqu'il est peu planifié et impulsif, catégorie qui affiche un plus faible taux de psychopathie.

\section{Types de victimes et psychopathie}

En ce qui concerne l'ensemble de leur criminalité, et plus particulièrement en ce qui a trait à l'homicide, les psychopathes s'attaquent davantage à des étrangers que les non-psychopathes (Williamson et al., 1987 ; Hersh et Gray-Little, 1998). Toutefois, les personnes proches sont aussi 
à risques avec les psychopathes. Notons aussi qu'il y a prédominance de victimes masculines chez les psychopathes, tandis que les femmes sont plus à risques avec les non-psychopathes (Williamson et al., 1987).

Cornell et al. (1996) ont comparé deux groupes de délinquants violents, l'un ayant exercé une violence instrumentale (dirigée vers un but précis) et l'autre ayant exercé une violence réactive (impulsive et émotive). Ils ont découvert que les individus du premier groupe avaient des scores à la PCL-R significativement plus élevés que ceux du deuxième groupe et qu'ils s'attaquaient plus fréquemment à des étrangers. Il est important de préciser que la différence est établie sur la base des scores totaux à la PCL-R et non sur la base des trois catégories diagnostiques (psychopathe, non-psychopathe et mixte). Ainsi, la prévalence de la psychopathie n'est pas établie comme telle. Il se peut donc qu'il existe une différence de moyenne à la PCL-R, mais qu'il ne se trouve pas plus de psychopathes dans un groupe que dans l'autre. Par ailleurs, ceux qui manifestent à la fois de la violence instrumentale et de la violence réactive sont inclus par Cornell et ses collaborateurs dans le groupe des agresseurs dits «instrumentaux». Or, les psychopathes, bien qu'utilisant majoritairement une violence instrumentale, peuvent aussi utiliser une violence réactive.

Un portrait unique du style de violence utilisée par le psychopathe ne peut donc pas être tracé. Même s'il y a prédominance de certains types de victimes et même s'il y a possibilité d'un mode réactionnel instrumental, il lui arrive fréquemment de se montrer imprévisible, tel que démontré par l'item 10 de la PCL-R mesurant la «faible maîtrise de soi», et l'item 14 mesurant l'«impulsivité». Le déficit émotionnel, caractérisé par le manque d'empathie (item 8 de la PCL-R) et l'affect superficiel (item 7), favorise un passage à l'acte contre les personnes avec qui l'agresseur a un lien d'intimité tout autant qu'envers les étrangers. Cela est soutenu par les observations de Brown et Forth (1997) qui ont étudié le lien entre la psychopathie, l'agression sexuelle et le type de victimes. Elles ont comparé deux groupes d'agresseurs sexuels, soit des psychopathes et des non-psychopathes. Les violeurs psychopathes ne présentaient pas plus de tension et de frustration, qui conduisent à une violence instrumentale, que de colère intense et de dépression sévère, qui résultent en une violence réactionnelle. La même constatation vaut aussi pour les non-psychopathes. De plus, elles n'ont relevé aucune différence significative du taux de psychopathie en fonction du lien avec la victime. Toutefois, ces données peuvent porter à confusion puisque 
la définition du lien avec la victime n'est pas clairement opérationnalisée. Il appert que les catégories ne sont pas exclusives, certains sujets pouvant se retrouver dans plus d'une catégorie de victimes. Par ailleurs, leur échantillon comprenant 60 cas, 16 se sont déclarés être étant dans une relation conjugale au moment du délit: c'est exactement le nombre d'agresseurs sexuels de conjointes retrouvé. Aucun agresseur de nonconjointe n'était en relation de couple avant le délit, ce qui pose le problème de la comparaison des groupes.

St-Yves et al. (document non publié), ont observé que les agresseurs sexuels ayant un lien d'intimité avec leur victime (conjointe, enfant, parenté) réagissent à des facteurs précurseurs de délits qui sont propres à la victime (par exemple, un conflit interpersonnel avec la partenaire ou une séparation). Ces derniers démontrent plus de colère, surtout durant le passage à l'acte, que les agresseurs sexuels qui n'ont pas de lien d'intimité. Ils ont aussi tendance à utiliser plus de force pour dominer et humilier leur victime, à exprimer moins de culpabilité, de honte et de remords, et à reconnaître moins de problèmes sexuels. Ces résultats s'apparentent à ceux de Groth et Birnbaum (1979) qui observent que les agresseurs sexuels de conjointes sont motivés par la colère.

Considérant que certains psychopathes peuvent s'en prendre autant à leur conjointe, à leurs proches qu'à des étrangers, que leur passage à l'acte peut être instrumental ou réactif, que leur motivation première, tout comme l'ensemble des violeurs conjugaux, n'est pas sexuelle, qu'ils se montrent insensibles au plan émotif (items 7 et 8 de la PCL-R), considérant également que l'étude de Brown et Forth (1997) présente certaines limites, il y a donc lieu de vérifier à nouveau l'hypothèse voulant que les caractéristiques des agresseurs sexuels de conjointes tiennent d'un mode de fonctionnement psychopathique par opposition aux agresseurs sexuels de femmes sans lien d'intimité.

\section{Méthodologie}

Sujets

L'échantillon est de 97 détenus dont 27 ont agressé sexuellement leur conjointe et 70 ont agressé sexuellement une femme adulte qui n'était pas leur conjointe. Il s'agit de tous les sujets répondant aux critères d'un programme de traitement pour délinquants sexuels ou pour conjoints violents, programmes offerts par le Service correctionnel du Canada 
(SCC) de la région du Québec. Un tel échantillon permet de réaliser une analyse de variance à deux groupes en assumant un effet de size large avec une puissance statistique de 0,80 pour un alpha de 0,05 (Cohen, 1960).

Le terme «conjointe» définit ici une femme avec qui le sujet entretient, ou a entretenu, une relation intime et sexuelle ${ }^{1}$. Nous avons considéré les données contenues dans les dossiers institutionnels (documents de la cour, informations du sujet et enquêtes communautaires) pour définir le lien entre l'agresseur et la victime. Les détenus qui ont agressé sexuellement leur conjointe ainsi qu'une autre personne n'ont pas été retenus afin d'assurer l'homogénéité des groupes.

Les deux groupes sont équivalents en ce qui a trait à l'âge. Les agresseurs sexuels de conjointes ont en moyenne 42,77 ans, avec un écarttype de 8,47 ; les agresseurs sexuels de non-conjointes ont en moyenne 40,21 ans, avec un écart-type de 8,71 (t $(94)=-1,29, \rho=$ n.s.). Les années de scolarisation sont aussi équivalentes. Les agresseurs sexuels de conjointes ont en moyenne 8,91 années d'étude, avec un écart-type de 2,61; les agresseurs sexuels de non-conjointes ont en moyenne 9,04 années d'étude avec un écart-type de 2,48 (t $(76)=, 20, \rho=$ n.s.).

Par ailleurs, le statut matrimonial au moment de l'enquête ne constitue pas une différence majeure, si ce n'est une tendance à avoir plus d'individus séparés ou divorcés chez les agresseurs de conjointes, ce qui est compréhensible considérant que la conjointe a été la victime par définition. Les données démontrent que les taux de sujets mariés ou en union libre au moment du délit sont similaires pour les deux groupes. Donc, ce n'est pas l'accessibilité à une conjointe potentielle qui est un élément déterminant dans le passage à l'acte.

\section{Instrument et procédure}

La version française de la PCL-R fut utilisée (Côté et Hodgins, 1996) pour établir la psychopathie. Dans la présente étude, l'accord interjuges est de .69 (71 sujets), ce qui peut être considéré comme substantiel (Landis et Koch, 1977). La cotation est faite auprès des détenus dans le

1. Cette définition est très proche de celle qu'utilisent les chercheurs (Straus et al., 1980; Gelles et Straus, 1988; Centre canadien de la statistique juridique, 1994), les organismes responsables des politiques (Comité canadien sur la violence faite aux femmes, 1993) et le Service correctionnel du Canada. 
cadre des activités régulières du Service correctionnel du Canada, par des professionnels sous supervision de psychologues. Toutes ces personnes ont été préalablement formées à l'utilisation de la PCL-R.

\section{Résultats}

Une trop faible proportion de cas diagnostiqués comme psychopathes à l'intérieur de l'échantillon global (3 sujets chez les agresseurs de conjointes et 2 sujets chez les agresseurs de non-conjointes) limite la possibilité d'effectuer des tests d'hypothèse. Le groupe des psychopathes est donc éliminé de l'analyse statistique basée dans un premier temps sur une conception taxinomique (catégorielle) de la psychopathie.

L'analyse effectuée à partir des deux seuls sous-groupes restant à la PCL-R, soit les «non-psychopathes» et ceux dits «mixtes», démontre qu'il existe une plus forte proportion de cas mixtes dans le groupe d'agresseurs sexuels de conjointes $(75 \%)$ que dans celui des agresseurs de non-conjointes $(42,6 \%)$; on observe que les non-psychopathes sont plus nombreux chez les agresseurs sexuels de non-conjointes $(57,4 \%)$ que chez les agresseurs sexuels de conjointes $(25 \%),\left(\chi^{2}(1, N=91)=\right.$ $7,43, \rho<, 01)$. Ces données montrent qu'il existe une relation entre la catégorie diagnostique à la PCL-R et le lien d'intimité qui unit l'agresseur et la victime.

TA B LE A U

Comparaison des agresseurs de conjointes et des agresseurs de non-conjointes au regard de la psychopathie

\begin{tabular}{|l|c|c|c|}
\hline PCL-R catégoriel (\%) & $\begin{array}{c}\text { Agresseurs de conjointes } \\
(\mathrm{n}=24)\end{array}$ & $\begin{array}{c}\text { Agresseurs de non-conjointes } \\
(\mathrm{n}=68)\end{array}$ & $\rho$ \\
\hline NP & $6(25 \%)$ & $39(57,4 \%)$ & \\
MIXTE & $18(75 \%)$ & $29(42,6 \%)$ & \\
\hline PCL-R continuum & Agresseurs de conjointes & Agresseurs de non-conjointes & \\
& $(\mathrm{n}=27)$ & $19=70)$ & $*$ \\
\hline M (é.-t.) & $22,81(7,07)$ & $7,00(3,35)$ & \\
\hline F 1 (Hare) & $8,44(3,64)$ & $8,88(4,20)$ & $* 2,25)$ \\
F 2 (Hare) & $10,15(4,28)$ & $2,59(1,70)$ & $*$ \\
F 1 (Cooke et al.) & $2,93(2,01)$ & $4,40(2,24)$ & \\
F 2 (Cooke et al.) & $5,52(2,10)$ & $5,06(2,62)$ & \\
F 3 (Cooke et al.) & $5,59(2,70)$ & & \\
\hline
\end{tabular}

* $\rho<0,01$

** $\rho<0,05$ 
Sur la base d'un continuum des résultats à la PCL-R, correspondant à une conception dimensionnelle de la psychopathie, les agresseurs sexuels de conjointes obtiennent un score moyen significativement plus élevé $(M=22,81$, é.-t. $=7,07)$ que les agresseurs sexuels de non-conjointes $(\mathrm{M}=19,16$, é.-t. $=6,25),(t(95)=2,49, \rho<, 05)$.

Par ailleurs, une analyse basée sur les deux facteurs recensés à la PCL-R n'a pas permis de faire ressortir de relations significatives entre l'un ou l'autre de ces facteurs et la nature du lien qui unit l'agresseur et la victime. Par contre, le modèle à trois facteurs a permis de faire ressortir que le facteur 2 (vie affective pauvre) distingue les deux groupes d'agresseurs sexuels, les agresseurs sexuels de conjointes $(M=5.52$, é.-t. $=2.10$ ) ayant une moyenne significativement plus élevée que les agresseurs sexuels de non-conjointes $(\mathrm{M}=4.40$, é.-t. $=2.24)$, ( $(95)=$ $-2.25, \rho<.05)$.

\section{Discussion}

Bien que l'hypothèse de départ ne puisse être maintenue, faute de sujets définis comme psychopathes, les analyses révèlent néanmoins des points intéressants. En interprétant les résultats à la PCL-R sous l'angle d'un continuum, les agresseurs sexuels de conjointes ont obtenu une moyenne plus élevée. Toutefois, il y a lieu de faire la différence entre cette donnée et l'utilisation de la PCL-R sous l'angle catégoriel. Ce faisant, on constate un taux plus élevé de non-psychopathes chez les agresseurs sexuels de non-conjointes, les cas mixtes étant plus nombreux chez les agresseurs sexuels de conjointes.

À la lumière des données antérieures sur la psychopathie et l'agression sexuelle, il est surprenant de trouver si peu de psychopathes dans cet échantillon, d'autant plus que la validation québécoise de l'échelle a démontré une concordance de la prévalence entre les échantillons du Québec et ceux de l'ensemble du Canada. L'aspect culturel ne semble donc pas être en cause ici (Hare, 1991). Quelques hypothèses pourraient être avancées pour expliquer cet état de fait. Il est possible que ces données représentent effectivement la réalité du phénomène étudié. Brown et Forth (1997) avaient obtenu 21 cas diagnostiqués psychopathes sur un échantillon de 60 délinquants sexuels. Ceci pourrait s'expliquer par l'ambiguité de la classification du lien avec la victime dans la recherche de Brown et Forth. Plusieurs de ces sujets avaient agressé tout autant leur conjointe qu'une personne qui leur était inconnue. Brown et Forth 
ont aussi établi trois liens distincts pour définir le lien unissant l'agresseur à sa victime, ajoutant la catégorie «connaissance». Nous avons préféré créer des classes plus hermétiques en éliminant un lien ambigu (connaissance) et ceux qui avaient agressé d'autres personnes que leur conjointe pour ce qui est des sujets du groupe des agresseurs de conjointes. Il se pourrait donc que les psychopathes se retrouvent dans cette catégorie de multiagresseurs, d'autant plus qu'il est reconnu que ces derniers font plus de victimes que les non-psychopathes (Hare et McPherson, 1984).

L'hypothèse d'une sélection préalable aux programmes est également à envisager pour expliquer le peu de psychopathes dans notre échantillon. En principe, tous les cas de délinquants sexuels et de conjoints violents sont orientés vers un programme de traitement par le Service correctionnel du Canada ${ }^{2}$. Ce n'est donc pas à l'étape de l'orientation qu'une éventuelle sélection pourrait s'opérer. De plus, comme le mentionne Hare (1991), les psychopathes sont habiles à se «prévaloir des structures et des programmes les plus susceptibles de donner l'impression qu'ils sont vraiment décidés à se réhabiliter». Donc, ils ne refuseront habituellement pas de participer, du moins une partie d'entre eux. Toutefois, cette motivation superficielle peut les amener à être exclus des groupes de traitement pour incapacité à véritablement se remettre en question et à changer. Ainsi, la relation entre la catégorie diagnostique de la PCL-R et le lien avec la victime d'agression sexuelle reste encore à explorer, notamment en recourant à un échantillon plus représentatif de l'ensemble des détenus ayant agressé sexuellement.

Un autre résultat intéressant est la moyenne plus élevée obtenue au facteur «vie affective pauvre» par les agresseurs sexuels de conjointes. Ce facteur, conçu par Cooke et Michie (2001), est inclus dans le facteur « un» du modèle original à deux facteurs (Hare, 1991). On se souviendra que, dans les études qui ont utilisé l'analyse factorielle originale, c'est le facteur un qui différencie la psychopathie de la personnalité antisociale. Il semble donc que ce résultat particulier révèle des caractéristiques centrales liées à la psychopathie chez les agresseurs sexuels de conjointes.

2. Les opinions et les conclusions n'engagent que les auteurs et non les divers organismes participants, notamment le Service correctionnel du Canada et le ministère de la Sécurité publique du Québec. 
L'originalité de la présente étude réside d'abord dans l'approche théorique du problème. En effet, très peu de recherches se sont penchées sur la spécificité de la population des conjoints sexuellement violents en comparaison avec d'autres dynamiques délictuelles, outre le lien qui les unit à leur victime. Dès lors, cette approche soulève la question de savoir si les violeurs conjugaux sont à comprendre sous l'angle de la délinquance sexuelle, de la violence familiale ou encore d'un fonctionnement typiquement psychopathique.

Brown et Forth (1997) sont celles qui se sont le plus rapprochées du présent sujet d'étude, mais l'ont fait uniquement sous l'angle de la délinquance sexuelle. Ainsi, elles ont sélectionné leurs sujets à partir du délit principal. Nous avons préféré sélectionner nos sujets à partir de programmes de traitement particuliers incluant un programme pour la violence conjugale. Ainsi, des sujets avec des délits officiels d'agressions sexuelles, mais aussi ceux qui avaient admis avoir agressé sexuellement leur conjointe sans avoir été reconnus coupables pour ce délit, étaient sélectionnés. Cela permet de réduire les problèmes de dénonciation liés à l'agression sexuelle d'une conjointe et d'avoir ainsi un échantillon d'agresseurs sexuels de conjointes plus représentatif de cette dynamique. Il faut penser que l'aspect non volontaire de la relation sexuelle entre conjoints peut faire problème dans la constitution de la preuve criminelle et ainsi entraîner un plus faible taux de dénonciations, d'accusations et de condamnations. Cet acte criminel n'est d'ailleurs reconnu que depuis 1983 au Canada (Roberts et Pires, 1992). Avant cette date, le conjoint pouvait faire l'objet d'accusations liées à la violence utilisée durant l'agression mais non quant à l'aspect sexuel du geste. Sous cet angle, il se pourrait que les sujets qui ont été condamnés pour agression sexuelle de leur conjointe correspondent aux cas les plus sévères d'agression, soit ceux qui suscitent une plus grande probabilité de dénonciation.

L'originalité de l'étude réside ensuite dans le fait que l'ensemble des diagnostics possibles à la PCL-R (psychopathe, mixte et nonpsychopathe) fut considéré. Brown et Forth n'avaient étudié que la différence psychopathes/non-psychopathes et démontraient qu'il n'y avait aucune relation statistique entre la psychopathie et le lien avec la victime d'agression sexuelle. L'ajout du diagnostic mixte apporte un éclairage différent. En effet, considérant la prévalence plus élevée d'agresseurs sexuels de conjointes chez les cas mixtes, il y a lieu de penser que ceux-ci seraient plus susceptibles de récidiver en commettant un délit possiblement sexuel. Dans le cadre d'un suivi d'une dizaine 
d'années, Wong (1996) a observé que, sur le plan de la récidive, les cas mixtes s'apparentaient davantage aux psychopathes, leurs taux de récidive générale étant similaires. Cette conclusion peut aussi se défendre sous l'angle d'un continuum. Les études sur la récidive et la psychopathie laissent entrevoir que le score à la PCL-R est un des meilleurs indicateurs de la récidive, violente et sexuelle (Harris et al., 1991; Quinsey et al., 1995; Quinsey et al., 1998; Seto et Barbarre, 1999). Cette donnée devra être prise en considération lors de l'évaluation du risque de ces délinquants.

Les limites de la recherche reposent principalement sur le dépistage des cas d'agresseurs sexuels de conjointes. Nous avons déjà parlé de la difficulté légale que représentent de tels dossiers, d'autant plus que les psychopathes sont plus opportunistes dans le passage à l'acte et utilisent à l'occasion un minimum de force pour subjuguer leur victime. Il se pourrait qu'il soit plus difficile pour les autorités judiciaires d'établir clairement la preuve de leur culpabilité. Un échantillon d'agresseurs sexuels de conjointes pris dès l'étape de la dénonciation permettrait de contourner en partie cette limite.

L'étude comporte certes ses limites, mais les différences observées, de même que les pistes de réflexion soulevées pour expliquer l'absence de psychopathes, ouvrent la voie à une poursuite de la recherche.

\section{Références}

American Psychiatric Association (1994). Diagnostic and statistical manual of mental disorders. (4 ed.). Washington, DC: American Psychiatric Association.

Barbaree, H., Seto, M., Serin, R., Amos, N., \& Preston, D. (1994). Comparisons between sexual and nonsexual rapist subtypes. Criminal Justice and Behavior, 21, 95-114.

Brown, S.L., \& Forth, A.E. (1997). Psychopathy and sexual assault: static risk factors, dynamic precursors, and rapist subtypes. Journal of Consulting and Clinical Psychology, 65, 848-857.

Caesar, P.L. (1986). Men who batter : a heterogeneous group. In L.K. Hamberger (ed.), The male batterer: characteristics of a heterogeneous population. Symposium conducted at the $94^{\text {th }}$ Annual Convention of the American Psychological Association, Washington, USA, août.

Centre canadien de la statistique juridique (1994). Résultats d'une enquête nationale sur l'agression contre la conjointe. Juristat, 14 (9), 1-22.

Cohen, J. (1960). A coefficient of agreement for nominal scales. Educational and Psychological Measurement, 20, 37-46. 
Comité canadien sur la violence faite aux femmes (1993). Un nouvel horizon: éliminer la violencce, atteindre l'égalité. Ottawa: Approvisionnements et Services Canada.

Cooke, D.J., Forth, A.E., \& Hare, R.D. (1998). Psychopathy: theory, research, and implications for society. Dordrecht, The Netherlands: Kluwer.

Cooke, D.J., \& Michie, C. (1997). An item response theory analysis of the Hare Psychopathy Checklist. Psychological Assessment, 9 (3), 3-13.

Cooke, D.J., \& Michie, C. (2001). Refining the construct of psychopathy: towards a hierarchical model. Psychological Assessment, 13 (2), 171-188.

Côté, G., \& Hodgins, S. (1996). L'Échelle de psychopathie de Hare-Révisée : éléments de la validation de la version française. Toronto: Multi-Health Systems.

Cornell, D.G., Warren, J., Hawk, G., Stafford, E., Oram, G., \& Pine, D. (1996). Psychopathy in instrumental and reactive violent offenders. Journal of Consulting and Clinical Psychology, 64, 783-790.

Flournoy, P.S., \& Wilson, G.L. (1991). Assesment of MMPI profiles of male batterers. Violence and Victims, 6, 309-320.

Gelles, R.J., \& Straus, M.A. (1988). Intimate violence: the causes and consequences of abuse in the American family. New York: Simon and Schuster.

Goldsmith, H.R. (1990). Men who abuse their spouses : an approach to assessing futur risk. Journal of Offender Counseling, Services and Rehabilitation, 15, 45-56.

Gondolf, E.W. (1988). Who are those guys? Toward a behavioral typology of batterers. Violence and Victims, 3, 187-203.

Gottman, J., Jacobson, N., Rushe, R., Shortt, J., Babcock, J., La Taillade, J., \& Waltx, J. (1995). The relationship between heart rate reactivity, emotionally aggressive behavior, and general violence in male batterers. Journal of Family Psychology, 9, 227-248.

Groth, A.N., \& Birnbaum, J. H. (1979). Men who rape: the psychology of the offender. New York: Plenum.

Hale, G., Zimostrad, S., Duckworth, J., \& Nicholas, D. (1988). Abusive partners: MMPI profiles of male batterers. Journal of Mental Health Counseling, 10, 214-224.

Hall, G.C.N., \& Hirschman, R. (1991). Toward a theory of sexual agression : a quadripartite model. Journal of Consulting and Clinical Psychology, 59, 662-669.

Hare, R.D. (1980). A research scale for the assessment of psychopathy in criminal populations. Personality and Individual Differences, 1, 111-119.

Hare, R.D. (1985). A comparison of procedures for the assessment of psychopathy. Journal of Consulting and Clinical Psychology, 53, 7-16.

Hare, R.D. (1991). The Hare Psychopathy Checklist - Revised. Toronto: MultiHealth Systems.

Hare, R.D. (1998). Psychopathy, affect, and behavior. In D.J. Cooke, A.E. Forth \& R.D. Hare (eds), Psychopatby: theory, research, and implications for society (105137). Dordrecht, The Netherlands: Kluwer.

Hare, R.D., \& McPherson, L.M. (1984). Violent and agressive behavior by criminal psychopaths. International Journal of Laws and Psychiatry, 7, 35-50. 
Harpur, T.J., Hakstian, R., \& Hare, R.D. (1988). Factor structure of the Psychopathy Checklist. Journal of Consulting and Clinical Psychology, 56, 741-747.

Harpur, T.J., Hare, R.D., \& Hakstian, R. (1989). A two-factor conceptualization of psychopathy: construct validity and implications for assessment. Psychological Assessment: A Journal of Consulting and Clinical Psychology, 1, 6-17.

Harris, G.T., Rice, M.E., \& Cormier, C.A. (1991). Psychopathy and violent recidivism. Law and Human Behavior, 15, 625-637.

Hart, S.D., \& Hare, R.D. (1998). Psychopathy: assessment and association with criminal conduct. In D.M. Stoff \& J. Maser (eds), Handbook of antisocial behavior (22-35). Toronto: Wiley.

Hersh, K., \& Gray-Little, B. (1998). Psychopathic traits and attitudes associated with self-reported sexual aggression in college men. Journal of Interpersonal Violence, 13, 465-471.

Holtzworth-Munroe, A., \& Stuart, G.L. (1994). Typologies of male batterers: three subtypes and the differences among them. Psychological Bulletin, 116, 476-497.

Huss, M.T., \& Langhinrichsen-Rohling, J. (2000). Identification of the psychopathic batterer: the clinical, legal, and policy implications. Aggression and Violent Behavior, A Review Journal, 5, 403-422.

Huss, M.T., Langhinrichsen-Rohling, J., \& Ramsey (1997). Can practitioners use our research: The clinical utility of batterer typologies. Presentation at the Association for the Advencement of Behavioral Therapy. Miami Beach, USA, novembre.

Koss, M.P., Dinero, T.E., Seibel, C.A., \& Cox, S.L. (1988). Stranger and acquaintance rape: are there differences in the victim's experience? Psychology of Women Quaterly, 12, 1-24.

Kropp, P.R., Hart, S.D., Webster C.D., \& Eaves D. (1995). The spousal assault risk assessment (SARA) guide. Vancouver: The British Columbia Institute on family violence.

Landis, J.R., \& Koch, G.G. (1977). The measurement of observer agreement for categorical data. Biometrics, 33, 159-174.

Prentky, R.A., \& Knight, R.A. (1991). Identifying critical dimensions for discriminating among rapists. Journal of Consulting and Clinical Psychology, 59, 643-661.

Quinsey, V.L., Harris, G.T., Rice, M.E., \& Cormier, C. (1998). Violent offenders: appraising and managing risk. Washington, USA: American Psychological Association.

Quinsey, V.L., Rice, M.E., \& Harris, G.T. (1995). Actuarial prediction of sexual recidivism. Journal of Interpersonal Violence, 10, 85-105.

Rice, M.E., Harris, G.T., \& Quinsey, V.L. (1990). A follow-up of rapists assessed in a maximum security psychiatric facility. Journal of Interpersonal Violence, 5 , 435-448.

Roberts, J. \& Pires, A.P. (1992). Le renvoi et la classification des infractions d'agression sexuelles. Criminologie, $X X V(1), 27-63$. 
Rochefort, S., \& Earls, C. (1998). Facteurs déterminants du fonctionnement antisocial et psychopathique chez deux groupes de sujets détenus en milieu carcéral. Présentation au $66^{\mathrm{e}}$ congrès de l'Association Canadienne française pour l'Avancement des Sciences (ACFAS), Québec, Canada, mai.

Rondeau, G. (1988). Les programmes québécois d'aide aux conjoints violents : rapport sur les seize organismes existants au Québec. Québec: Ministère de la Santé et des Services Sociaux.

Saunders, D.G. (1992). A typology of men who batter women: three types derived from cluster analysis. American Journal of Orthopsychiatry, 62, 264275.

Serin, R.C. (1996). Violent recidivism in criminal psychopaths. Law and Human Behavior, 20, 207-217.

Serin, R.C., Malcolm, P.B., Khanna, A., \& Barbaree, H.E. (1994). Psychopathy and deviant sexual arousal in incarcerated sexual offenders. Journal of Interpersonal Violence, 9, 3-11.

Seto, M.C., \& Barbaree, H.E. (1999). Psychopathy, treatment behavior, and sex offenders recidivism. Journal of Interpersonal Violence, 14, 1235-1248.

Sonkin, D.J. (1987). The assessment of court-mandated male batterers. In D.J. Sonkin (ed.), Domestic violence on trial: psychological and legal dimensions of family violence (174-196). New York: Springer.

Stewart, L., Hill, J., \& Cripps, J. (1999). Le traitement en violence familiale dans les milieux correctionnels. In L.L. Motiuk \& R.C. Serin (eds), Compendium 2000 des programmes correctionnels efficaces (97-110). Ottawa: Service correctionnel du Canada.

Straus, M.A., Gelles, J.R., \& Steinmetz, S. (1980). Behind closed doors: violence in the American family. New York: Doubleday Anchor Press.

Stuart, E.P., \& Campbell, J.C. (1989). Assessment of patterns of dangerousness with battered women. Issues in Mental Health Nursing, 10, 245-260.

Walker, L.E. (1979). The battered woman. New York: Harper \& Row.

Widiger, T.A. (1998). Psychopathy and normal personality. In D.J. Cooke, A.E. Forth \& R.D. Hare (eds), Psychopatby: theory, research and implications for society (47-68). Dordrecht, The Netherlands: Kluwer.

Williamson, S.E., Hare, R.D., \& Wong, S. (1987). Violence: criminal psychopaths and their victims. Canadian Journal of Behavioral Science, 19, 454-462.

Wong, S. (1996). Recidivism and the criminal career of psychopaths: a longitudinal study. In D.J. Cooke, A.E. Forth, J.P. Newman \& R.D. Hare (eds), Issues in criminological and legal psychology: $N^{0} 24$, International perspectives on psychopathy (147-152). Leicester, UK: British Psychological Society. 